\title{
Búsqueda activa de casos de leishmaniasis visceral zoonótica en población infantil indígena y canina colombiana
}

\author{
An active search for cases of zoonotic visceral leishmaniasis in \\ indigenous Colombian children and the canine population
}

\author{
Marlyn H. Romero ${ }^{1}$, Myriam C. López ${ }^{2}$ y Jorge A. Sanchez ${ }^{1}$
}

\begin{abstract}
1 Departamento de Salud Animal, Facultad de Ciencias Agropecuarias, Universidad de Caldas. Maniza-les, Colombia.marlyn.romero@ucaldas.edu.co,jorge.sanchez@ucaldas.edu.co

2 Departamento de Salud Pública, Facultad de Medicina, Universidad Nacional de Colombia, Bogotá. mclopezp@unal.edu.co
\end{abstract}

Recibido 14 Abril 2009/Enviado para Modificación 24 Octubre 2009/Aceptado 4 Noviembre 2009

\section{RESUMEN}

Objetivos Realizar la búsqueda activa de casos humanos y caninos de leishmaniasis visceral zoonótica (LVZ) mediante la determinación de anticuerpos IgG contra Leishmania infantum por la técnica de Inmunofluorescencia indirecta (IFAT) en una zona endémica colombiana y evaluar factores de riesgo relacionados con la presentación de la enfermedad.

Métodos Un total de 580 niños indígenas menores de cinco años y 270 perros criollos localizados en cinco veredas del municipio de Coyaima (Tolima), fueron evaluaos por medio de la determinación de anticuerpos, usando como antígeno la cepa colombiana de Leishmania infantum (infantum) MHOM/COL/CL044B. Así mismo, se aplicó una encuesta a 527 viviendas del área de estudio para evaluar factores de riesgo y medidas de protección.

Resultados No se presentó seroreactividad de la población infantil ante antígenos totales de $L$. infantum por la prueba IFAT. La frecuencia de anticuerpos anti IgG contra $L$. infantum en caninos fue del 31,5\% (185/270). Se detectaron deficiencias en las condiciones de saneamiento básico de las viviendas. Se evidenció el uso de toldillos impregnados con insecticidas en el $48 \%$ (130/270) de las viviendas, pobre conocimiento de la enfermedad y baja demanda de los servicios médicos tradicio-nales $(45,1 \%)$.

Conclusiones Teniendo en cuenta la importancia de los caninos en el ciclo de transmisión de la LVZ es necesario fortalecer las medidas de control para interrumpir la cadena de transmisión reservorio-vector-humano, evaluar las estrategias de pro-moción en hábitos protectores desarrolladas por las autoridades sanitarias y mejorar las condiciones de vida y ambientales de la población susceptible.

Palabras Clave: Leishmania, diagnóstico serológico, Colombia (fuente: DeCS, BIREME). 


\begin{abstract}
Objectives Carrying out an active search for cases of canine and human zoonotic visceral leishmaniasis (LVZ) by determining IgG antibodies against Leishmania infantum by indirect immunofluorescence (IFAT) and assessing the risk factors associated with the disease occurring in a Colombian endemic area.

Methods 580 indigenous children aged less than five and 270 cross-bred dogs from 5 rural areas near the town of Coyaima (Tolima) were evaluated by determining their antibodies using the Colombian Leishmania infantum (infantum) MOHOM/ COL/CLO44B strain as antigen. 527 households in the area were sur-veyed to assess the risk factors and protective measures being taken.

Results No sero reactivity to Leishmania infantum antigens by IFAT test was observed in the child population. Leishmania infantum antibodies were observed in $68.5 \%$ of the dogs (185/270). The survey found deficiencies in the housing's sanitary conditions. The use of in-secticide-impregnated bed-nets was evident in $48 \%(130 / 270)$ of the dwellings, as were poor knowledge of the disease and a low demand for traditional medical services (41.5\%).

Conclusions Control measures must be strengthened for interrupting the reservoir-vector-human transmission chain, evaluating health authorities' strategies for promoting protective habits and improving living conditions and the susceptible population's envi-ronment.
\end{abstract}

Key Words: Canine visceral leishmaniasis, serological test, Colombia (source: $\mathrm{MeSH}, \mathrm{NLM}$ ).

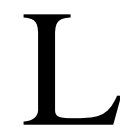

a Leishmaniasis Visceral Zoonótica (LVZ) en América es una enfermedad sistémica causada por parásitos del género Leishmania infantum $(=L$. cha-gasi) y transmitida por la picadura de insectos flebotomíneos del género Lutzomyia (Lu.) longipalpis y esporádicamente Lu. evansi en América (1-3). La LVZ es endémica en Colombia, pero el mayor número de casos se pre-sentan en los departamentos de Córdoba, Sucre, Bolívar, Tolima y Huila $(2,4)$. Los reservorios de mayor importancia son Didelphis marsupialis (chu-cha, fara, runcho) y Canis familiares $(2,4,5)$. La enfermedad afecta princi-palmente a niños menores de 5 años y a pacientes inmunosuprimidos $(3,6,7)$, produciendo una letalidad del $95 \%$ cuando no es tratada a tiempo $(6,7)$.

Las medidas de control de la LVZ están basadas en cuatro estrategias: búsqueda activa de casos, tratamiento oportuno, control de los reservorios domésticos y control antivectorial $(6,8)$. Se ha demostrado que la detección activa de casos en áreas endémicas es una estrategia muy valiosa porque disminuye las tasas de morbi-mortalidad y de discapacidad en niños suscep-tibles $(6,9)$. Sin embargo, el diagnóstico de la leishmaniasis humana es difí-cil. Los principales síntomas son indistinguibles de otras enfermedades para-sitarias como malaria y síndromes asociados con hepato y esplenomegalia $(10,11)$. La 
confirmación del diagnóstico se debe realizar por examen para-sitológico, el cual es un método invasivo y requiere de experticia del profesional (12). El aislamiento de los parásitos es costoso y difícil y las técnicas moleculares no tienen mucha aplicación en condiciones de campo $(11,13)$. Debido a estas limitaciones las técnicas serológicas se convierten en una herramienta de tamizaje importante para ser aplicada en poblaciones huma-nas y animales susceptibles. En el presente trabajo se plantearon como obje-tivos presentar los resultados de la detección activa de casos de LVZ en niños indígenas menores de cinco años del municipio de Coyaima (Tolima) por medio de la técnica Inmunofluorescencia indirecta (IFAT), evaluar fac-tores de riesgo relacionados con la enfermedad y establecer la reactividad de sueros caninos ante antígeno total de $L$. infantum por IFAT.

\section{MÉTODOS}

Área geográfica

El municipio de Coyaima está localizado en el sur del Departamento del Tolima, con un área de $667 \mathrm{~km}^{2}$, una temperatura promedio de $28,2{ }^{\circ} \mathrm{C}$ y con una precipitación anual de $1502 \mathrm{~mm}$ (14). Se seleccionaron las veredas Santa Martha Diamante, Totarco Tamarindo, Doyare Porvenir, Totarco Dinde y Agua Fría, donde se han presentado los mayores reportes de casos de LVZ en el municipio.

Muestras de suero humanas

Con el apoyo del Grupo de Control Social de la Leishmaniasis visceral (LV) de la Secretaría Departamental de Salud del Tolima, se concertaron reuniones de sensibilización y concertación con los coordinadores de los resguardos indígenas de las áreas involucradas en el trabajo. Se procedió a obtener el consentimiento informado aprobado por el comité de bioética de la Se-cretaria Departamental de Salud de la Gobernación del Tolima por parte de madres de los infantes participantes. Se tomaron $2 \mathrm{ml}$ de sangre venosa pe-riférica de quinientos ochenta niños indígenas menores de cinco años $(\mathrm{n}=580)$, se centrifugaron, se distribuyeron en alicotas y se almacenaron a $-21{ }^{\circ} \mathrm{C}$ hasta su procesamiento.

Muestras de suero caninas

Se analizaron doscientos setenta sueros caninos $(n=270)$ recolectados du-rante el año 2006. Se obtuvo el consentimiento informado de los propietarios de los animales para realizar la toma de muestras de sangre, siguiendo las normas éticas para el manejo de animales establecidas en la Ley 84 de 1989 y las normas éticas para investigación sin riesgos en comunidades, descritas en la resolución del Ministerio de Salud No. 8430 de 1993. 
Encuesta

Se aplicó una encuesta casa a casa a 527 viviendas localizadas en el área rural del área de estudio donde se evaluaron variables relacionadas con la presentación de la enfermedad.

Inmunofluorescencia Indirecta (IFAT)

La técnica fue desarrollada siguiendo la metodología descrita por Corredor (1). Como antígeno se emplearon promastigotes en fase metacíclica de Leishmania infantum (infantum) cepa MHOM/COL/CL-044B. Se considera-ron como títulos positivos aquellos iguales o superiores a 1:32 para la pobla-ción infantil y canina.

\section{RESULTADOS}

Población infantil

El 31,7 \% de la población infantil menor de cinco años evaluada correspon-dió al sexo masculino (184/580) y el 68,3\% restante (396/580) al femenino. La totalidad de los sueros analizados no fueron reactivos a la prueba IFAT.

Tabla 1. Factores potencialmente asociados con la presencia de anticuerpos anti-Leishmania infantum en cinco veredas del municipio de Coyaima, Tolima, 2006

\begin{tabular}{lcc}
\hline \multirow{2}{*}{ Variable } & \multicolumn{2}{c}{ Frecuencia } \\
& $n$ & $\%$ \\
\hline Infraestructura sanitaria de viviendas & & \\
$\quad$ Deficiente & 50 & 83.3 \\
Aceptable & 45 & 8.8 \\
$\quad$ Buena & & \\
Conocimiento de la enfemedad (transm isión y medidas & & \\
de control) & 63 & 11,1 \\
$\quad$ Conoce & 507 & 88,9 \\
$\quad$ No conoce & & \\
Uso de toldillo & 297 & 52,1 \\
$\quad$ No lo usan & 207 & 36,3 \\
La familia & 66 & 11.6 \\
Sólo los niños & & \\
Presencia de caninos & 399 & 70 \\
Si & 171 & 30 \\
No & & \\
Consulta médica tradicional & 257 & 45,1 \\
$\quad$ Si & 313 & 54,9 \\
$\quad$ No &
\end{tabular}

Población canina

La población estudiada estuvo conformada por perros mestizos $(n=270)$, con una edad promedio de 3 años. El 55,5 \% machos y el 44,5 \% hembras. La seroreactividad ante antígenos totales de L. infantum en el grupo fue del 31,5\% (185/270) por IFAT. 
Encuesta

El $66 \%$ (376/570) -de las viviendas de la zona endémica evaluadas presentan paredes en barro o bahareque y con pisos en tierra en el 31,5\% (180/570) de los casos. A pesar de que el 57,31\% (327/570) de la población posee un abastecimiento de agua potable procedente del acueducto, el 42,7\% (243/570) restante se abastece de aljibes o pozos. La disposición de excretas y de residuos sólidos se realiza a campo abierto en el 41,3\% (235/570) y el 77, $6 \%$ (335/570) de las viviendas respectivamente. En la Tabla 1 se pre-sentan los resultados del análisis de otras variables evaluadas en el área de estudio.

\section{DISCUSION}

La LVZ es la forma clínica más grave de las leishmaniasis, porque puede ser fatal en la población infantil si no se administra tratamiento oportunamente, teniendo en cuenta que las infecciones bacterianas secundarias complican el cuadro clínico (15), siendo por lo tanto importante efectuar un diagnóstico precoz como medida de prevención secundaria $(8,11)$. En Colombia el dia-gnóstico serológico de $L$. infantum en humanos y caninos se realiza con la técnica IFAT $(1,16)$.

En la población infantil evaluada no se presentó seroreactividad ante antí-geno total de L. infantum, resultados que difieren con los reportados por Corredor (1), quien encontró una seropositividad en población indígena de diferentes edades del 5,1 \% y 4,6 \% mediante las técnicas IFAT e Inmuno-ensayo ligado a Enzimas (ELISA) respectivamente. Sin embargo, la población canina evaluada que convivía en las viviendas con los infantes presentó una seroreactividad alta del 31,5\% a IFAT, resultados que concuerdan con los reportados por Romero (16) en la zona endémica del sur del Tolima, pero superiores a los encontrados por Fernández (17) en el departamento del Huila y por Zerpa (6) en Venezuela.

Los caninos domésticos han sido descritos como importantes reservorios en el ciclo de transmisión de L. infantum en América Latina, incluyendo Colombia $(6,16,18)$, por su alta susceptibilidad a la infección y parasitismo cutáneo intenso $(16,19)$, y principalmente por su estrecha relación con el hombre. Se considera como un factor de riesgo la presencia de caninos se-ropositivos para la infección por $L$. infantum en población humana $(8,11,18,20)$. Teniendo en cuenta los resultados obtenidos, se podría sugerir que las medidas de control en esta zona endémica podrían fortalecerse mediante el control de la sobrepoblación canina y la selección de estrategias que inte-rrumpan la transmisión reservorio-vectorhumano $(9,16,18,19)$. 
Entre las medidas recomendadas para el control la LVZ se encuentra la identificación de caninos positivos y su posterior eutanasia $(9,20)$. La efectividad de esta medida se encuentra en controversia, porque algunos investigadores han reportado un limitado impacto en la reducción de la incidencia de la enfermedad en población humana y canina $(21,22)$. Sin embargo, otros estudios indican que la infección de los animales precede a la aparición de casos humanos y que la tasa de infección en estos últimos se incrementa en las áreas con una alta prevalencia de la infección canina en donde el vector está presente (9). Lo mismo sucede con el uso de insecticidas tópicos o collares impregnados con estos, porque se reportan trabajos en donde su adopción ha permitido la disminución del reporte de casos nuevos en población infantil y canina $(23,24)$, cuando han sido implementados durante un año. Sin embargo, otros estudios han referido ineficiencia de esta medida de control, por el alto número de collares perdidos durante el estudio (20).

La evaluación de las condiciones socio-económicas del área de estudio pone de manifiesto un nivel bajo. Estudios observacionales que han inferido sobre la asociación de estas condiciones con el riesgo de la LVZ, han reportado que tienen una relación inversamente proporcional (12). En la zona endémica las entidades de salud pública han instaurado como medidas de control la entrega gratuita de toldillos impregnados con insecticidas como factor de protección para la LVZ. De acuerdo con los resultados presentados en la Tabla 1, podríamos sugerir que en la población evaluada ha tenido buen impacto esta medida, porque el $48 \%$ de las viviendas encuestadas lo usan. Estudios efectuados en Nepal e India (3) han demostrado su eficacia en la disminución de la incidencia de leishmaniasis visceral humana, sin embargo ésta se ve afectada por los hábitos de sueño de la población y por los hábitos de picadura de los vectores $(3,12)$.

Se puede asumir que el conocimiento por parte de la población humana de la severidad y el potencial impacto de LVZ podría favorecer la implementación de medidas preventivas. Investigadores han encontrado fuerte evidencia de asociación entre el conocimiento sobre la enfermedad y la disminución de la incidencia de la LVZ (12). En las veredas evaluadas se evidenció desconocimiento sobre estos aspectos (Tabla 1), a pesar que dentro de las medidas de prevención instauradas se encuentra la promoción sobre hábitos protectores de LVZ, las cuales deben ser evaluadas por las autoridades sanitarias, de acuerdo con los resultados encontrados en el trabajo.

Se pudo observar además que existe una alta proporción de la población indígena encuestada $(54,9 \%)$ que no demanda los servicios de la consulta médica 
tradicional, aspecto relevante a tener en cuenta por la dificultad de atender e iniciar tratamiento oportunamente a los pacientes de LVZ, teniendo en cuenta que su diagnóstico clínico requiere de experiencia y de soporte de laboratorio, porque los signos clínicos no son patognomónicos $(11,15,25)$. Es primordial que en el área de estudio se implementen métodos sensibles y específicos para el diagnóstico rápido como la prueba con tira reactiva re-combinante $\mathrm{K} 39$, la prueba de aglutinación directa con antígeno criodese-cado y la prueba de aglutinación con látex para detectar el antígenoen la orina $(6,9,11)$, que fortalezcan la vigilancia epidemiológica activa y el trata-miento temprano de los casos.

La información recolectada sobre la epidemiología de la LVZ en población infantil y canina es importante para la definición y evaluación de las medidas de control implementadas por las autoridades de salud pública del departa-mento del Tolima. Siendo necesario desarrollar nuevas estrategias de prevención orientadas no sólo a interrumpir el ciclo de transmisión del parásito, sino también a proveer condiciones sanitarias y ambientales a las viviendas, mejorar las condiciones de saneamiento básico y garantizar la seguridad alimentaria a la población infantil susceptible. Así mismo, es prioritario fortalecer las actividades de promoción en hábitos protectores a la comunidad

Agradecimientos: A la Secretaría Departamental de Salud del Tolima, Colombia, por el financiamiento de esta investigación.

\section{REFERENCIAS}

1. Corredor A, Alvarez CA, Agudelo CA, Bueno M, López MC, Cáceres E, Reyes P, Duque S, Gualdrón LE, Santacruz M. Prevalence of Trypanosoma cruzi and Leishmania chagasi infection and risk factors in a Colombian indigenous population. Rev. Inst.Med. trop. Sao Paulo 1999; 41(4):229-234.

2. Travi BL, Tabares CJ, Cadena H, Ferro C, Osorio Y. Canine visceral leishmaniasis in Colom-bia: relationship between clinical and parasitologic status and infectivity for sand flies. Am J Trop Med Hyg. 2001; 64:119-24.

3. Chappuis F, Sundar S, Hailu A, Ghalib H, Suman R, Peeling RW; Alvar J; Boelaert M. Vis-ceral leishmaniasis: What are the needs for diagnosis, treatment and control?. Nature Re-views/ Microbiol 2007; 5:873-882.

4. Corredor A, Gallego J, Tesh RB, Morales A, Ferro C, Young D G, Kreutzer R D, Boshell J, Palau M T, Cáceres E, Peláez D. Epidemiology of visceral leishmaniasis in Colombia. Am J Trop Med Hyg. 1989; 5:480-486.

5. Travi B, Ferro C, Cadena H, Montoya-Lerma J, Adler GH. Canine Visceral Leishmaniasis: dog infectivity to sand flies from non-endemic areas. Res Vet Sci 2002; 72:83-86.

6. Zerpa O, Ulrich M, Borges R, Rodríguez V, Centeno M, Negrón E, Belizario D, Convit J. Epidemiological aspects of human and canine visceral leishmaniasis in Venezuela. Pan Am J Public Health 2003; 13(4):239-245. 
7. Cascio A, Calattini S, Colomba C, Scalamogna Ch, Galazzi M, Pizzuto M, Camilli R, Gramic-cia M, Titone L, Corbellino M, Antinori S. Polymerase chain reaction in the diagnosis and prog-nosis of Mediterranean visceral leishmaniasis in immunocompetent children. Pediatrics 2002; 9(2):1-5.

8. Flores MD, Postigo JR, Mendoza NM, Cruz I, Alvar J, Bastrenta B. Leishmaniasis visceral subclínica en 123 individuos de un cantón de la provincia Caranavi-La Paz. Arch Pediatr Urug 2004; 75(4):338-344.

9. Maia-Elkhoury ANS, Alves W, Soua-Gomes ML, de Sena JM, Luna EA. Visceral leishmania-sis in Brazil: trends and challenges. Cad. Saúde Pública 2008; 24(12):2941-2947.

10. De Medeiros FS, Tavares-Neto J, D'Oliveira Jr. A. Alteraciones hepáticas en la leishmania-sis visceral (kalazar) en niños: revisión sistemática de la literatura. Acta Gastroentol Latinoam. 2007; 37(3):150-157.

11. Terán-Angel G, Schallig $\mathrm{H}$, Zerpa O, Rodríguez V, Ulrich M, Cabrera M. The direct aggluti-nation test as an alternative method for the diagnosis of canine and human visceral leishmania-sis. Biomédica 2007; 27:447-53

12. Kolaczinski JH, Reithinger R, Worku DT, Ocheng A, Kasimiro J, Kabatereine N, Brooker S. Risk Factors of visceral leishmaniasis in east Africa: a case-control study in Pokot territory of Kenya and Uganda. Int J Epidemiol 2008; 37(2):344-352.

13. Guerin PJ, Oliaro P, Sundar S, Boelaert M, Croft MB, Desjeux P, Wasunna MK, Bryceson DM. Visceral leishmaniasis: current status of control, diagnosis, and treatment, and a proposed research and development agenda. The Lancet Infec Dis 2002; 2:494-500.

14. Instituto Colombiano Agustín Codazzi. Diccionario Geográfico de Colombia. Bogotá D.C.; 1996.

15. Bhattacharya SK, Sur D, Karbwang J. Childhood visceral leishmaniasis. Indian J Med Res 2006; 123:353-356.

16. Romero MH, López MC, Echeverry MC, Rivas F. Leishmaniasis visceral canina: pruebas diagnósticas no identifican estados reales de la infección. Rev. Salud pública 2008; 10(2):290-298.

17. Fernández J, Bello F, López MC, Moncada LI, Vargas JJ, Ayala MS, Nicholls RS, Lozano CA. Seroprevalencia de la leishmaniasis visceral canina en la comuna 8 de Neiva y en cuatro municipios del Huila, Colombia. Biomédica 2006; 26(Supl. 1):121-130.

18. Dantas-Torres F. The role of dogs as reservoirs of Leishmania parasites, with emphasis on Leishmania (leishmania) infantum and Leishmania (Viannia) braziliensis. Vet Parasitol. 2007; 149:139-146.

19. Courtenay O, Rupert J, Quinnell L, Garcez M, Shaw J, Dye D. Infectiousness in a Cohort of Brazilian dogs: Why culling fails to control visceral leishmaniasis in areas of high transmission. J Infect Dis 2002; 186:1314-20.

20. Dantas-Torres F, Brandao-Filho SP. Visceral leishmaniasis in Brazil: revisiting paradigms of epidemiology and control. Rev Inst Med Trop Sao Paulo 2006; 48:151-6.

21. Palatnik-De-Sousa CB, Wania R, Franca-Silva JC, Da Costa RT, Reis BA, Palatnik M, Mayrink W, Genaro O. Impact of canine control on the epidemiology of canine and human visceral Leishmaniasis in Brazil. The Am Soc Trop. Med Hyg. 2001; 65(5):510-517.

22. Ashford D, David Jr, Freire M, David R, Sherlock I, Eulalio MDC, Sampaio DP, Badaro R. Studies on Control of Visceral Leishmaniasis: Impact of dog Control on canine and human visceral Leishmaniasis in Jacobina, Bahía, Brazil. Am.J.Trop. Med. Hyg. 1998; 59(1):53-57.

23. Reithinger R, Coleman PG, Alexander B, Vieira EP, Assis G, Davies CR. Are insecticide-impregnated dog collars a feasible alternative to dog culling as a strat-egy for controlling canine visceral leishmaniasis in Brazil? Int. J. Parasitol. 2004; 34:55-62.

24. David JR, Stamm LM. Bezerra HS, Souza RN, Killick-Kendrick R, Oliveira JM. Deltamethrinimpregnated dog collars have a potent anti-feeding and insecticidal effect on Lutzomyia longipalpis and Lutzomyia migonei. Mem. Inst. Oswaldo Cruz 2001; 96:839-847.

25. Altaf Ch, Ahmed P, Ashraf T, Anwar M, Ahmed I. Clinicopathological features of childhood visceral leishmaniasis in Azad Jammu \& Kashmir Pakistan. J Ayub Med Coll Abbottabad 2005; 17(4):1-4. 